\title{
Explosive Magnetorotational Instability in Keplerian Disks
}

\author{
Yu. Shtemler * Liverts $\oplus$ and M. Mond \\ Department of Mechanical Engineering, \\ Ben-Gurion University of the Negev \\ Beer-Sheva 84105, Israel \\ (Dated: October 11, 2018)
}

\begin{abstract}
Differentially rotating disks under the effect of axial magnetic field are prone to a nonlinear explosive magnetorotational instability (EMRI). The dynamic equations that govern the temporal evolution of the amplitudes of three weakly-detuned resonantly interacting modes are derived. As distinct from exponential growth in the strict resonance triads EMRI occurs due to the resonant interactions of a MRI mode with stable Alfvén-Coriolis and magnetosonic modes. Numerical solutions of the dynamic equations for amplitudes of a triad indicate that two types of perturbations behavior can be excited for resonance conditions: (i) EMRI which leads to infinite values of the three amplitudes within a finite time, and (ii) bounded irregular oscillations of all three amplitudes. Asymptotic explicit solutions of the dynamic equations are obtained for EMRI regimes and are shown to match the numerical solutions near the explosion time.
\end{abstract}

PACS numbers: PACS 98.62.Mw

\section{INTRODUCTION}

The magnetorotational instability (MRI, [1] - 3]) is believed to play a key role in the angular momentum transfer in accretion disks, and has been thoroughly investigated through linear analysis as well as nonlinear magnetohydrodynamic (MHD) simulations under a wide range of conditions and applications. First attempts to achieve analytical insight into the nonlinear evolution of the MRI focused on the dissipative saturation of the instability $([4]-[5])$ in environments that are characteristic of laboratory experiments.

Recently it has been suggested that the mechanism of dissipationless wave interaction plays an important role in the nonlinear development of the MRI in astrophysical disks $([6]-9])$. According to one such scenario the MRI forms a triad of interacting modes with a stable slow or fast Alfvén-Coriolis (AC) mode and a stable magnetosonic (MS) mode. This is a generalisation and adaptation to the thin, rotating, axially stratified disks of the well known three-wave interaction in static, homogeneous and infinite plasmas and fluids $([10]-11])$. The saturation of the MRI by non-resonant excitation of a MS wave, as well as by resonantly exciting two smallamplitude linearly stable modes have been investigated for astrophysical disks $([\underline{6}]-[9])$. It has been shown that in the resonance case with zero frequency mismatch the two linearly stable modes are either bounded or grow exponentially due to the nonlinear coupling to the saturated MRI. In the case of zero frequency mismatch is named hereafter strict resonance in order to distinct it

*Electronic address: shtemler@bgu.ac.il

${ }^{\dagger}$ Electronic address: eliverts@bgu.ac.il

‡Electronic address: mond@bgu.ac.il on the general resonance case of small nonzero frequency mismatch. As is also well known for different dispersive plasma media, nonlinear interactions of three resonant modes may give rise to explosive instability (EI), under which the amplitudes of the triad modes grow to infinity in finite time $($ 12] -20$]$ ).

The current work is focussed on the evolution of resonant triads in thin Keplerian disks. In particular, it is shown that the exponential nonlinear instability of the strict resonance triads is replaced by explosive MRI (EMRI) in resonance conditions with small non zero frequency mismatch.

\section{THE PHYSICAL MODEL}

Nonlinear evolution of the axisymmetric MRI in spatially stratified rotating plasma is studied for Keplerian disks threaded by a poloidal magnetic field. The thin disk approximation ([21]-22]) is applied to the MHD equations by ignoring viscosity, electrical resistivity, and radiation effects. This approximation developed in [7]- 8] for strict resonance interactions in thin Keplerian disks is applied in the present study to detuned resonance triads. Hereby the main results of these studies are summarized in that section:

\section{A. Governing relations}

Assuming the disk to be vertically isothermal yields the following dimensionless dynamic system:

$$
\begin{gathered}
\frac{D \mathbf{V}}{D t}=-\bar{C}_{S}^{2} \frac{\nabla n}{n}+\frac{1}{\beta_{*}} \frac{\mathbf{j} \times \mathbf{B}}{n}-\epsilon^{-3} \nabla \Phi, \\
\frac{\partial n}{\partial t}+\nabla \cdot(n \mathbf{V})=0
\end{gathered}
$$




$$
\begin{gathered}
\frac{\partial \mathbf{B}}{\partial t}+\nabla \times \mathbf{E}=0, \quad \mathbf{E}=-\mathbf{V} \times \mathbf{B}, \\
\mathbf{j}=\nabla \times \mathbf{B}, \quad \nabla \cdot \mathbf{B}=0, \\
B_{r}=B_{\theta}=0, n=0 \text { for } z= \pm \infty .
\end{gathered}
$$

The assumption of vertically isothermal disk implies that $\nabla P=\bar{C}_{S}^{2} \nabla n$, where $\bar{C}_{S}^{2}=\bar{T}(r)$ is the dimensionless sound speed; $T=\bar{T}(r)$ is the steady-state background temperature; $P(r, z, t), n(r, z, t), \mathbf{V}$ and $\Phi(r, z)$ are the total plasma pressure, number density, plasma velocity and gravitational potential due to the central body; $\{r, \theta, z\}$ are the cylindrical coordinates; $t$ is time; $D / D t=\partial / \partial t+(\mathbf{V} \cdot \nabla)$ is the material derivative; $\mathbf{B}, \mathbf{j}$ and $\mathbf{E}$ are the magnetic field, current density and electric field, respectively.

A MHD model of dissipationless quasi-neutral plasmas is characterized by three dimensional velocities calculated at the characteristic radius, $r_{*}$, namely, the Keplerian rotation, $V_{K *}=r_{*} \Omega_{*} \equiv \sqrt{G M_{c} / r_{*}}$, the sound velocity, $C_{S_{*}}=\sqrt{T_{*} / m_{i}}$, and the Alfvén velocity, $V_{A *}=$ $B_{*} / \sqrt{4 \pi m_{i} n_{i}}$, which produce two dimensionless parameters: the plasma beta $\beta_{*}$ and the Mach number $M_{*}$ that is equals to the inverse small disk aspect ratio $\epsilon=H_{*} / r_{*}$

$$
\beta_{*}=4 \pi \frac{P_{*}}{B_{*}^{2}} \equiv \frac{C_{S *}^{2}}{V_{A *}^{2}}, \quad M_{*}=\frac{V_{K *}}{C_{S *}} \equiv \epsilon^{-1} .
$$

The dimensional values $M_{c}, G, m_{i}, n_{i}$ and $T_{*}, B_{*}, H_{*}=$ $C_{S *} / \Omega_{*}$ are as follows: the mass of the central body, the gravitational constant, the ion mass and number density, and the temperature, poloidal magnetic field and the disk height calculated at $r=r_{*}$.

\section{B. Steady-state}

The smallness of $\epsilon$ makes it useful to define a slow radial coordinate, and in addition to leading order in $\epsilon$, it is convenient to replace the independent variables by the following self-similar quantities:

$$
\xi=\epsilon r \sim \epsilon^{0}, \tau=\bar{\Omega}(\epsilon r) t \sim \epsilon^{0}, \eta=z / \bar{H}(\epsilon r) \sim \epsilon^{0} .
$$

The radial and axial coordinates $\xi$ and $\eta$ are stretched in compliance with assumption of small disk aspect ratio; $\bar{H}(\xi)=\bar{C}_{s}(\xi) / \bar{\Omega}(\xi)$ is the semi thickness of the disk. The Keplerian steady-state configuration is described to leading order in $\epsilon$ by the equilibrium conditions in the radial and axial directions as follows:

$$
\begin{gathered}
\Phi(r, z)=-\frac{1}{\sqrt{r^{2}+z^{2}}} \equiv-\epsilon \bar{\Phi}(\xi)+\frac{\epsilon^{3}}{2} \bar{C}_{S}^{2}(\xi) \bar{\psi}(\eta)+O\left(\epsilon^{5}\right) \\
n(r, z)=\epsilon^{0} \bar{N}(\xi) \bar{n}(\eta)+O\left(\epsilon^{2}\right)
\end{gathered}
$$

$$
\begin{gathered}
V_{r}=V_{z}=0, \quad V_{\theta}=\epsilon^{-1} \bar{V}(\xi)+O(\epsilon), \\
B_{r}=B_{\theta}=0, \quad B_{z}=\epsilon^{0} \bar{B}(\xi),
\end{gathered}
$$

where $\bar{C}_{S}(\xi), \bar{B}(\xi), \bar{N}(\xi)$ are arbitrary functions;

$\bar{V}(\xi) \equiv \xi \bar{\Omega}(\xi)=\frac{1}{\sqrt{\xi}}, \bar{\Phi}(\xi)=\frac{1}{\xi}, \bar{\psi}(\eta)=\eta^{2}, \bar{n}(\eta)=e^{-\eta^{2} / 2}$.

\section{Nonlinear perturbations}

Both steady-state equilibrium as well as the perturbed variables are scaled with well-defined powers of the small parameter $\epsilon$ :

$$
F(r, z, t)=\epsilon^{\bar{S}} \bar{F}(\xi, \eta ; \epsilon)+\epsilon^{S^{\prime}} F^{\prime}(\xi, \eta, \tau ; \epsilon) .
$$

Here $F$ stands for any dependent variable, the bar and the prime denote equilibrium and perturbed variables, respectively, which are characterized by gauge functions $\epsilon^{\bar{S}}$ and $\epsilon^{S^{\prime}}$. The various values of $\bar{S}$ are determined above in eqs. (8), while the values of $S^{\prime}$ are obtained to be zero for all the dependent variables except for the perturbed axial magnetic field for which $S^{\prime}=1$.

The perturbed dependent variables can now be scaled with the following slow-radius varying functions:

$$
\mathbf{v}=\mathbf{V}^{\prime} / \overline{\mathbf{C}}_{\mathbf{S}}(\xi), \quad \nu=\mathbf{n}^{\prime} / \overline{\mathbf{N}}(\xi), \quad \mathbf{b}=\mathbf{B}^{\prime} / \overline{\mathbf{B}}(\xi) .
$$

Then inserting Eqs. (7) - (10) in Eqs. (1)-(5), keeping the leading order terms in $\epsilon$ and eliminating $v_{r}$ and $v_{\theta}$ yield the following dimensionless dynamic equations:

$$
\frac{\partial^{2} b_{r}}{\partial \tau^{2}}-2 \frac{\partial b_{\theta}}{\partial \tau}-\frac{1}{\beta(\xi)} \frac{\partial}{\partial \eta}\left[\frac{1}{\bar{n}(\eta)} \frac{\partial b_{r}}{\partial \eta}\right]-3 b_{r}=N_{r}
$$

$$
\begin{gathered}
\frac{\partial^{2} b_{\theta}}{\partial \tau^{2}}+2 \frac{\partial b_{r}}{\partial \tau}-\frac{1}{\beta(\xi)} \frac{\partial}{\partial \eta}\left[\frac{1}{\bar{n}(\eta)} \frac{\partial b_{\theta}}{\partial \eta}\right]=N_{\theta}, \\
\frac{\partial^{2} \nu}{\partial \tau^{2}}-\frac{\partial}{\partial \eta}\left[\bar{n}(\eta) \frac{\partial}{\partial \eta}\left(\frac{\nu}{\bar{n}(\eta)}\right)\right]=N, \\
b_{r}=b_{\theta}=0, \quad \nu=0 \text { for } \eta= \pm \infty .
\end{gathered}
$$

Explicit expressions for the nonlinear righthand sides $N_{r}$, $N_{\theta}$ and $N$ in eqs. (11) - 113) as well as the equations for $v_{r}, v_{\theta}$ and $v_{z}$ that relate them to $b_{r}, b_{\theta}$ and $\nu$ are presented in [8]. The equation for the perturbed axial magnetic field decouples from the rest of the equations and drops out from the governing system. To leading order in $\epsilon$ the radial derivatives also drop from the resulting system without approximation of frozen radial variable, and the radial dependence enters only through the local plasma beta: $\beta(\xi)=\beta_{*} \bar{N}(\xi) \bar{C}_{s}^{2}(\xi) / \bar{B}^{2}(\xi)$. 


\section{Linear perturbations}

Modifying the mass density profile to $\bar{n}(\eta)=\operatorname{sech}^{2} \eta$ enables the analytical solution of the linearized set of equations (11) -(14) for small perturbations. The resulting linear eigenmodes are decoupled into two families of the AC and MS modes ([8] and references therein). The eigenfunctions of $\mathrm{AC}$ modes for the in-plane perturbed velocities are expressed in terms of the Legendre polynomials $P_{k}(\zeta)(k=1,2, \ldots)$ of $\zeta=\tanh (\eta)$. The AC family represents in-plane perturbations and forms a discrete spectrum whose eigenvalues are:

$$
\omega_{k, l}= \pm \sqrt{\frac{\beta+6 \beta_{k}+l \sqrt{\left(\beta+6 \beta_{k}\right)^{2}-36 \beta_{k}\left(\beta_{k}-\beta\right)}}{2 \beta}} .
$$

Here $\omega_{k, l}$ are labeled by two integers: $-\infty<k<\infty$ that plays the role of the axial wave number, and $l=-1(+1)$ that represents the slow (fast) AC modes. The fast $\mathrm{AC}$ modes are stable while the slow AC modes may become unstable. The number of unstable slow AC modes is determined by the local plasma beta $\beta(\xi)$. Thus, the threshold for exciting $k$ unstable modes is given by $\beta_{k}=k(k+1) / 3, k=1,2, \ldots$ It is those unstable slow $\mathrm{AC}$ modes that constitute the MRI whose eigenvalues are given by $\omega_{m,-1}=i \gamma, m=1, \ldots, k$. Of particular importance is the fact that for $\beta=\beta_{k}, \gamma_{k}=0$ is a double root of the dispersion equation. The second family of eigen-oscillations in thin Keplerian disks is the vertical MS modes. The latter are stable, possess a continuous spectrum, and their eigenfunctions are expressed in terms of special functions [6]. The AC and MS families of the linear eigenmodes are the building blocks of the present nonlinear analysis be unfolded in the next sections.

\section{NONLINEAR DYNAMICS OF DETUNED TRIADS}

\section{A. Scenario of three-mode nonlinear interaction}

The scenario that is introduced in the current work is a generalization of the mechanism described in 7] - 8] for the strict resonance triads: A $\beta$ value slightly above the first threshold $\beta_{1}=2 / 3$ for MRI is considered. As a results there is only one unstable MRI mode, that is characterized by axial wave number $k=1$. A MRI eigenmode that is characterized by complex frequency $0+i \gamma$ forms a triad of interacting modes with a stable fast or slow AC mode and a stable MS wave that are characterized by a real frequency $\omega_{a} \equiv \omega_{k, l}$ and $\omega_{s}$, respectively. The condition for the occurrence of resonant interaction between the three eigenmodes is $\omega_{s}=\omega_{a}+\Delta \omega$, where the frequency mismatch $\Delta \omega$ is much smaller than each of the eigenfrequencies $\omega_{a}$ and $\omega_{s}$. The resonance condition is easily satisfied due to the continuous nature of the
MS spectrum. This condition can be satisfied also if the Gaussian mass distribution is considered. In that case the MS spectrum is discrete, however, as was pointed out in [8], for any frequency $\omega_{k, l}$ of a stable AC mode, a corresponding eigenfrequency of the Gaussian MS spectrum may be found such that the resonance condition is satisfied. The customary resonance condition on the axial wave number is not needed here due to the axial stratification of the mass density; it is replaced by the solvability conditions of the higher orders boundary value problem in the axial coordinate. The resonance condition on the radial wave number drops out in the thin disk approximation according to which the radial derivatives are negligible. The three-wave interaction is a direct result of the influence of the perturbed in-plane magnetic pressure gradients on the acoustic modes, and the simultaneous axial convection of the AC modes by the acoustic perturbations.

The main goal of the current section is to derive a set of coupled ordinary differential equations that govern the evolution of the amplitudes of the modes that take part in the resonant interaction. During the linear stage those amplitudes are constants that are determined by the initial conditions. However as the resonant interaction gains in importance, the mutual interaction changes dramatically their time evolution. Thus, while the equations that govern the amplitudes of the stable AC and MS modes are similar to their first order classical counterparts [1], the equation for the amplitude of the MRI is of second order, reflecting the multiplicity two of the eigenvalue at the threshold beta [8].

Deriving the amplitude equations starts with observing that for small values of the growth rate $\gamma$ and frequency mismatch $\Delta \omega \sim \gamma$, the resonant interactions are described by two distinct time scales: a fast time $\bar{\tau}=t$, and a slow time $\tilde{\tau}=\gamma t$. Assuming further that the frequency mismatch $\Delta \omega$ is of order $\gamma$, each of the perturbations due to the AC modes $(f(\eta, t))$ as well as those due to the MS waves $(g(\eta, t))$ may be represented as a sum of zeroth and first harmonic terms in the fast time (higher harmonics are neglected), each with an amplitude varying with the slow time:

$$
f(\eta, t)=f_{0}(\eta, \tilde{\tau})+\left[f_{1}(\eta, \tilde{\tau}) e^{-i \omega_{a} \bar{\tau}}+c . c\right],
$$

where the subscripts 0 or 1 denote the harmonic number. A similar expression is written also for $g(\eta, t)$. Contributions to $f_{0}$, the zeroth harmonic $\mathrm{AC}$ perturbations, come from the MRI, a non-resonant excitation of a zeroth harmonic MS wave, and the interaction of the stable fast $\mathrm{AC}$ and the MS eigenmodes, all of which may be represented as:

$$
\begin{aligned}
f_{0}(\eta, \tilde{\tau}) & =A_{0}(\tilde{\tau}) \Psi_{0}(\eta)+A_{0}(\tilde{\tau}) H_{0}(\tilde{\tau}) \psi_{0,0}(\eta) \\
& +\left[A_{1}^{*}(\tilde{\tau}) H_{1}(\tilde{\tau}) \psi_{-1,1}(\eta)+c . c\right],
\end{aligned}
$$

where $A_{0}$ is the real-valued amplitude of the MRI $\left(e^{ \pm \tilde{\tau}}\right.$ during the linear stage), $\Psi_{0}$ its linear eigenfunction, 
$A_{1}, H_{1}$ are the amplitudes of the stable $\mathrm{AC}$ and $\mathrm{MS}$ modes, respectively (constants during the linear stage), $H_{0}$ is the amplitude of the non-resonantly excited MS wave, and $\psi_{-1,1}, \psi_{0,0}$ are yet to be determined coupling functions. In a similar manner the expressions for $f_{1}, g_{1}$ are given by:

$$
\begin{aligned}
& f_{1}(\eta, \tilde{\tau})=A_{1}(\tilde{\tau}) \Psi_{1}(\eta)+\left[A_{0}(\tilde{\tau}) H_{1}(\tilde{\tau}) \psi_{0,1}(\eta)+c . c .\right] \\
& g_{1}(\eta, \tilde{\tau})=H_{1}(\tilde{\tau}) \Phi_{1}(\eta)+\left[A_{0}(\tilde{\tau}) A_{1}(\tilde{\tau}) \phi_{0,1}(\eta)+c . c .\right]
\end{aligned}
$$

where $\Psi_{1}, \Phi_{1}$ are the eigenfunctions of the AC and MS modes, respectively, $\phi_{0,1}\left(\psi_{0,1}\right)$ is the coupling function between the MRI and the $\mathrm{AC}(\mathrm{MS})$ mode. The first terms on the right hand sides of eqs. (17)-(19) describe the three linear modes that participate in the resonant interaction. The amplitude of the non resonantly driven MS wave may be shown to be $H_{0}(\tilde{\tau})=A_{0}^{2}(\tilde{\tau})[8]$.

\section{B. Nonlinear equations for triad amplitudes}

The amplitudes $A_{0}, A_{1}$, and $H_{1}$ are the main players in the current work and deriving the set of ordinary differential equations that govern their slow-time dynamic evolution is the main concern of this section. Thus, recalling the second order degeneracy of the MRI eigenfrequency at the threshold beta eq. (15)), the dynamic equation for $A_{0}$ is necessarily of second order, that in view of its resonance interaction with the two other modes is conjectured to be given by:

$$
\gamma^{2} \frac{d^{2} A_{0}}{d \tilde{\tau}^{2}}=\gamma^{2} A_{0}+\Gamma_{0,0} A_{0}^{3}+\left[\Gamma_{-1,1} A_{1}^{*} H_{1} e^{-i \delta \omega \tilde{\tau}}+c . c\right] .
$$

The corresponding first order equations for $A_{1}$ and $H_{1}$ are identical with their classical counterparts [11]:

$$
\gamma \frac{d A_{1}}{d \tilde{\tau}}=i \Gamma_{0,1} A_{0} H_{1} e^{-i \delta \omega \tilde{\tau}}, \gamma \frac{d H_{1}}{d \tilde{\tau}}=i \Gamma_{1,0} A_{0} A_{1} e^{i \delta \omega \tilde{\tau}} .
$$

Here $\delta \omega=\Delta \omega / \gamma \sim \gamma^{0}$, and $\Gamma_{i, j} \sim \gamma^{0}$ are nonlinear coupling coefficients yet to be determined that measure the interaction between the various waves. A clear hierarchy emerges from the principle of least degeneracy of eqs. (20)-(21) according to which the amplitude of the MRI mode in thin Keplerian disks is much larger than amplitudes of the rest of two stable modes $A_{0} \sim \gamma \gg A_{1} \sim H_{1} \sim \gamma^{3 / 2}$. As a result, all terms in eqs. (20)-(21) are of the same order in $\gamma$ as distinct from classical dispersive plasma media with $A_{0} \sim A_{1} \sim H_{1} \sim \gamma$, where the self-excitation cubic term in eq. (20) is neglected as compared with the resonant quadratic term.

Equations (16)-(21) are inserted now into the MHD equations, and solved order by order in $\gamma$. As expected, the lowest order reproduces the linear results for the eigenfunctions $\Psi_{0}(\eta), \Psi_{1}(\eta)$ and $\Phi_{1}(\eta)$ in relations (17)(19). The next order yields non homogeneous linear ordinary differential equations in $\eta$ that are subject to zero boundary conditions at the disk edges for the following four nonlinear coupling functions: (i) zeroth MRI harmonics $\psi_{0,0}(\eta)$ and $\psi_{-1,1}(\eta)$; (ii) first $\mathrm{AC}$ harmonic $\psi_{0,1}(\eta)$, and (iii) first MS harmonic $\phi_{0,1}(\eta)$. Since the associate homogeneous problems for $\psi_{0,0}(\eta), \psi_{-1,1}(\eta)$, $\psi_{0,1}(\eta)$ and $\phi_{0,1}(\eta)$ coincide with the eigenvalue problems for $\Psi_{0}(\eta), \Psi_{1}(\eta)$ and $\Phi_{1}(\eta)$, respectively, solutions of the corresponding non homogeneous problems have singularity. To avoid that singularities the four coupling coefficients $\left(\Gamma_{0,0}, \Gamma_{-1,1}, \Gamma_{0,1}, \Gamma_{1,0}\right)$ are introduced into eqs. (20)-(21) and are determined from the solvability conditions for the non homogeneous problems for $\psi_{0,0}(\eta), \psi_{-1,1}(\eta), \psi_{0,1}(\eta), \phi_{0,1}(\eta)$. The resulting values of $\left(\Gamma_{0,0}, \Gamma_{-1,1}, \Gamma_{0,1}, \Gamma_{1,0}\right)$ are exactly the same as for the strict resonance system [8]. The difference between the two systems is only in the exponential factors $e^{ \pm i \delta \omega \tilde{\tau}}$ in eqs. (20)-(21) that describe the effect of small mismatch on the evolution of resonant triads.

Finally, by appropriately rescaling the amplitudes eqs. (20)-(21) may be recast in the following form:

$$
\begin{gathered}
\frac{d^{2} a_{0}}{d \tilde{\tau}^{2}}=a_{0}-a_{0}^{3}+\sigma_{0} \alpha^{2}\left[a_{1}^{*} h_{1} e^{-i \delta \omega \tilde{\tau}}+c . c\right] \\
\frac{d a_{1}}{d \tilde{\tau}}=i \alpha a_{0} h_{1} e^{-i \delta \omega \tilde{\tau}}, \frac{d h_{1}}{d \tilde{\tau}}=i \sigma_{1} \alpha a_{0} a_{1} e^{i \delta \omega \tilde{\tau}}
\end{gathered}
$$

where $a_{0} \sim a_{1} \sim h_{1} \sim \gamma^{0}$ are the rescaled amplitudes $A_{0}, A_{1}, H_{1}$, respectively; $\alpha=\sqrt{\left|\Gamma_{0,1} \Gamma_{1,0} / \Gamma_{0,0}\right|}$, $\sigma_{0}=\operatorname{sign}\left(\Gamma_{0,1} \Gamma_{-1,1}\right)$, and $\sigma_{1}=\operatorname{sign}\left(\Gamma_{0,1} \Gamma_{1,0}\right)$. Equations (22)-(23) constitute the dynamic system that is investigated in the next section. Each interacting triad is thus characterized by a set of three coefficients $\alpha, \sigma_{0}$ and $\sigma_{1}$ which determines the nature of its dynamic evolution. The MRI amplitude in thin disks is governed by a Duffing equation of second order in contrast to the first order equation for classical dispersive plasma media and fluids.

\section{The generalized Manley-Rowe relations}

A convenient representation of the amplitudes of the $\mathrm{AC}$ and $\mathrm{MS}$ modes is given by $a_{1}(\tilde{\tau})=$ $\rho_{a}(\tilde{\tau}) e^{-i \varphi_{a}(\tilde{\tau})}, h_{1}(\tilde{\tau})=\rho_{h}(\tilde{\tau}) e^{-i \varphi_{h}(\tilde{\tau})}$ (recall that $a_{0}$ is real-valued). In terms of these variables equations (22)(23) give rise to the following two constants of motion:

$$
\begin{aligned}
& E_{0}=\frac{1}{2}\left(\frac{d a_{0}}{d \tilde{\tau}}\right)^{2}-\frac{1}{2}\left(a_{0}^{2}-\frac{1}{2} a_{0}^{4}\right)+\mathcal{E}, \\
& E_{1}=\frac{1}{2} \rho_{h}^{2}+\sigma_{1} \frac{1}{2} \rho_{a}^{2} .
\end{aligned}
$$

Here $\mathcal{E}=\frac{1}{2} \sigma_{0} \alpha \delta \omega\left(\sigma_{1} \rho_{h}^{2}-\rho_{a}^{2}\right)-2 \sigma_{0} \alpha^{2} a_{0} \rho_{a} \rho_{h} \cos \varphi, \varphi=$ $\varphi_{a}-\varphi_{h}-\delta \omega \tilde{\tau}$, and $E_{0}$ and $E_{1}$ are constants. Equations (24) and (25) are the Manley-Rowe type relations 
that are generalized for the amplitude of the MRI mode governed by the Duffing equation of the second order.

\section{SOLUTIONS FOR TRIAD AMPLITUDES}

The solutions of eqs. (22)-(23) exhibit much more complex behavior than their strict-resonance counterparts. As is demonstrated in 7], 8] resonant triads with $\delta \omega=0$ are divided into stable $\left(\sigma_{1}=+1\right)$ and unstable $\left(\sigma_{1}=-1\right)$ triads. In the unstable case the MRI mode saturates by nonlinearly coupling to AC and MS modes that grow exponentially, while all the three amplitudes within stable triads are bounded and exhibit oscillatory behavior. As observed in Table 1 triads are stable $\left(S_{k, l}\right)$ if the role of the stable $\mathrm{AC}$ wave is played by a fast $\mathrm{AC}$ mode, and unstable $\left(U_{k, l}\right)$ for slow AC waves. This classification is inherited from the strict resonance case, however, the exponential growth of amplitudes is changed by explosive one for detuned resonance triads. Table 1 presents the coefficients of systems (20)-(21) and (22)-(23) for seven (out of infinitely many) triads composed of MS, fast or slow AC modes, and a background MRI mode $(k=1, l=-1)$ at its threshold beta $\beta_{1}=2 / 3$. The triad stability criteria that emerge from Table 1 are that $\sigma_{1}=1>0$ is necessary and sufficient while $\sigma_{1}=-1<0$ is necessary for EMRI (also for unstable triads $\sigma_{0}=-1$ ). This will be demonstrated below explicitly and numerically.

\section{A. Unstable triads $\left(\sigma_{1}=-1\right)$}

Solving eqs. (22)-(23) numerically indicate that unlike the strict-resonance case, all three modes may grow explosively in time, namely, the solutions tend to infinity within a finite time, which is termed the explosion time and denoted by $\tilde{\tau}_{e}$. To simplify the calculations, the particular case $\rho(\tilde{\tau}) \equiv \rho_{a}(\tilde{\tau})=\rho_{h}(\tilde{\tau})$ is considered:

$$
\begin{aligned}
& \frac{d^{2} a_{0}}{d \tilde{\tau}^{2}}=a_{0}-a_{0}^{3}+2 \sigma_{0} \alpha^{2} \rho^{2} \cos \phi \\
& \frac{d \rho}{d \tilde{\tau}}=-\alpha a_{0} \rho \sin \phi, \frac{d \phi}{d \tilde{\tau}}=-2 \alpha a_{0} \cos \phi-\delta \omega
\end{aligned}
$$

As follows from the forced Duffing equation (26) for the MRI amplitude the force term exhibits temporal oscillations instead of being a constant in the strict resonance case. Numerical solutions demonstrate that for such triads, as $\tilde{\tau} \rightarrow \tilde{\tau}_{e}, a_{0}$ and $\rho \rightarrow \infty$, while $\varphi \rightarrow$ $(2 M+1) \pi / 2+\varphi_{e}$ where $M$ is an integer and $\varphi_{e} \rightarrow 0$, such that $a_{0} \varphi_{e}$ remains finite. These observations lead to the ansatz $a_{0}(\tilde{\tau}) \varphi_{e}(\tilde{\tau})=$ Const that results in the following asymptotic expressions close to $\tilde{\tau}_{e}$ :

$$
\hat{a}_{0} \simeq \frac{K_{0}}{1-\hat{\tau}}, \quad \hat{\rho} \simeq \frac{K_{1}}{(1-\hat{\tau})^{2}}, \quad \hat{\varphi} \simeq K_{2}(1-\hat{\tau}),
$$

where $K_{0}=2(-1)^{M+1}, K_{1}^{2}=\operatorname{sign}(\delta \omega)\left(6+24 / \alpha^{2}\right)$, $K_{2}=-1 / 3 ; \hat{\tau} \equiv \tilde{\tau} / \tilde{\tau}_{e} ; \hat{a}_{0}, \hat{\rho}$ and $\hat{\varphi}$ are the normalized amplitudes and phase, respectively. It is evident that under the ansatz explosive solutions exist only for $\delta \omega>0$. With that restriction asymptotic expressions (27) in the blow up vicinity are universal in the sense that they are independent of both $\delta \omega$ and $\tilde{\tau}_{e}$. Thus $\tilde{\tau}_{e}$ cannot be calculated by the analysis that leads to eqs. (27) and is determined by the initial conditions. Although $\delta \omega>0$ in eqs. (27), numerical solutions demonstrate that explosive solutions exist also for $\delta \omega<0$, for which $a_{0}(\tilde{\tau}) \varphi_{e}(\tilde{\tau})$ is not a constant near $\tilde{\tau}_{e}$ as it required in eqs. (27).

Asymptotic expansions of eqs. (22)-(23) or (26) in the artificial large parameter $\alpha$ yield that $\alpha \tilde{\tau}$ and $\delta \omega / \alpha$ are the natural scales for slow time and frequency mismatch. Thus neglecting the first two terms in the righthand side of the first of eqs. (26) yields $a_{0}, \rho$ and $\phi$ for unstable triads that are uniformly valid up to $\alpha^{-2}$ in the whole range of $\alpha$ in Table 1. In particular this implies that the principal unstable triad, $U_{2,-1}$, has the biggest among other unstable triads value of $\alpha=20.5$, and hence the shortest $\tilde{\tau}_{e}$. In this sense that triad is deemed the most unstable, and hence is the mostly used for the numerical demonstration in the following.

Figure 1 presents a comparison between the numerical solution of eqs. (26) and the universal explosive solutions (27). It is seen that after an initial transient period, as time approaches the explosive time, the two dashed lines, which represent the numerical solutions of eqs. (26) for two different values of $\delta \omega / \alpha$, converge to the full line, which represents solution of eqs. (27).
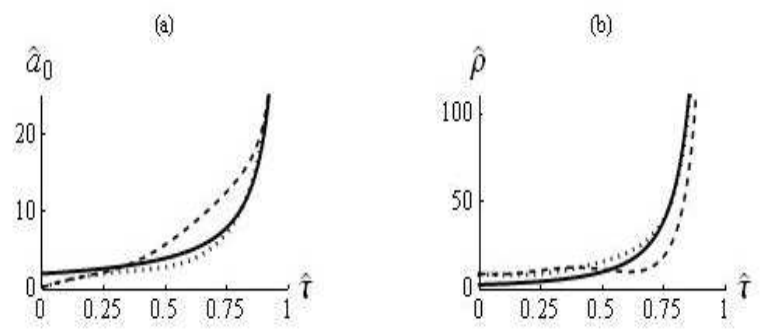

FIG. 1: Comparison of the numerical solution of eqs. (26) with universal explosive solutions (27) for the principal unstable triad $U_{2,-1}(\alpha=20.5)$. Full line: solution of eqs. (27) with $\delta \omega / \alpha=1$. Lower and upper dashed lines: solution of eqs. (26) with $\delta \omega / \alpha=1$ and 2.5 , respectively, in the partial initial $\operatorname{data}\left(\tilde{\tau}=0: a_{0}=0.05, d a_{0} / d \tilde{\tau}=0.5 \alpha, \rho=0.4, \phi=\pi / 4\right)$.

The non-normalized amplitudes of the unstable triad, as obtained from the numerical solution eqs. (26), are depicted in Fig. 2 for typical value of the frequency mismatch $\delta \omega / \alpha=0.5$. Note that the negative sign of $\sigma_{1}$ enables the unbounded growth of the amplitudes of the participating modes while keeping $E_{1}$ constant according to the Manley-Rowe relation (25). It is interesting to note that while $A_{0} \sim \gamma /\left(\tilde{\tau}-\tilde{\tau}_{e}\right)$ and $A_{1} \sim$ 
TABLE I: Eigen-frequencies $\omega_{k, l}\left(\beta_{1}\right)$ of the AC modes and nonlinear coupling coefficients of systems (20)-(21) and (22) - (23) for resonant triads composed of the stable MS and fast $(l=1)$ or slow $l=1)$ AC modes $(k=1,2,3, \ldots)$, and the background MRI mode $(k=1, l=-1)$ at its threshold beta $\beta_{1}=2 / 3 ; k$ is the axial wavenumber; $l$ is the indicator of fast or slow AC mode. $S_{k, l}$ and $U_{k, l}$ are stable and unstable triads for fast and slow AC modes. The stabilizing non-resonant coupling coefficient $\Gamma_{0,0}=-27 / 35$ [] ].

\begin{tabular}{cccccccc} 
Triad & \multicolumn{2}{c}{ Stable Unstable } & Stable & Unstable Stable Unstable & Stable \\
$\left\{S_{k, l}, U_{k, l}\right\}$ & $S_{1,1}$ & $U_{2,-1}$ & $S_{2,1}$ & $U_{3,-1}$ & $S_{3,1}$ & $U_{4,-1}$ & $S_{4,1}$ \\
\hline$k$ & 1 & 2 & 2 & 3 & 3 & 4 & 4 \\
\hline$l$ & 1 & -1 & 1 & -1 & 1 & -1 & 1 \\
\hline$\beta_{k}$ & $2 / 3$ & 2 & 2 & 4 & 4 & 15 & 15 \\
\hline$\omega_{k, l}\left(\beta_{1}\right)$ & $\sqrt{7}$ & $\approx \sqrt{7 / 2}$ & $\approx \sqrt{31 / 2}$ & $\sqrt{10}$ & $\sqrt{27}$ & $\approx \sqrt{39 / 2}$ & $\approx \sqrt{83 / 2}$ \\
\hline$\Gamma_{1,0}$ & -0.68 & -3.40 & -5.06 & 2.01 & 1.81 & 16.46 & 13.81 \\
\hline$\Gamma_{0,1}$ & -0.20 & 94.97 & -0.03 & -4.40 & 0.11 & -0.44 & 0.011 \\
\hline$\Gamma_{-1,1}$ & -0.14 & -2.16 & 0.026 & 3.78 & 0.66 & 1.66 & -0.01 \\
\hline$\alpha=\sqrt{\left|\frac{\Gamma_{0,1} \Gamma_{1,0}}{\Gamma_{0,0}}\right|}$ & 0.42 & 20.5 & 0.44 & 3.4 & 0.51 & 3.07 & 0.44 \\
\hline$\sigma_{0}=\operatorname{sign}\left(\Gamma_{0,1} \Gamma_{-1,1}\right)$ & +1 & -1 & -1 & -1 & +1 & -1 & -1 \\
\hline$\sigma_{1}=\operatorname{sign}\left(\Gamma_{1,0} \Gamma_{0,1}\right)$ & +1 & -1 & +1 & -1 & +1 & -1 & +1
\end{tabular}

(a)

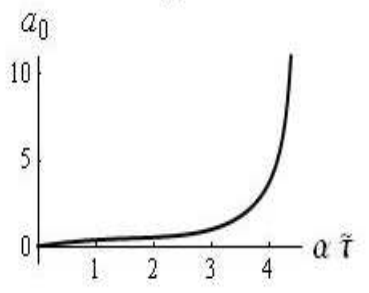

FIG. 2: Solution of eqs. (26) in the partial initial data $(\tilde{\tau}=0$ : $\left.a_{0}=0.05, d a_{0} / d \tilde{\tau}=0.5 \alpha, \rho=0.4, \phi=\pi / 4\right)$ for the principal unstable triad $U_{2,-1}\left(\alpha=20.5, \delta \omega / \alpha=0.5, \alpha \tilde{\tau}_{e} \approx 4.5\right)$.

$H_{1} \sim \gamma^{3 / 2} /\left(\tilde{\tau}-\tilde{\tau}_{e}\right)^{2}$ the amplitude of MRI, initially much larger, grows much slower in the blow up vicinity than the other of two modes, the amplitudes become comparable when $\left(\tilde{\tau}-\tilde{\tau}_{e}\right) \sim \gamma^{1 / 2}$.

Since the blow-up will occur earlier for the shortest $\tilde{\tau}_{e}$ its value is an effective measure of the nonlinear instability. Since strict resonance unstable triads $(\delta \omega \equiv 0)$ grow exponentially they have $\tilde{\tau}_{e}=\infty$, while detuned resonance triads have rather bounded values $\tilde{\tau}_{e}$, when $\delta \omega$ approaches \pm 0 (Fig. 3), except of the vicinity of the mismatch threshold value. Indeed, Fig. 3 for two different sets of the initial data demonstrates that dependence of $\tilde{\tau}_{e}$ vs. $\delta \omega$ is strongly influenced by the initial data. Although EMRI occurs for any $\delta \omega>0$ with the shortest value $\tilde{\tau}_{e}$ of about 0.2 for both sets of the initial data, for $\delta \omega<0$ EMRI occurs only when $\delta \omega$ does not exceed the threshold given by $\left|\delta \omega_{t h}\right| / \alpha$ (that value significantly depends on initial data), otherwise the amplitudes of the triad oscillate. This also implies that in the limit of vanishing frequency-mismatch the triad's amplitudes do not convergence to those in the strict resonance case.

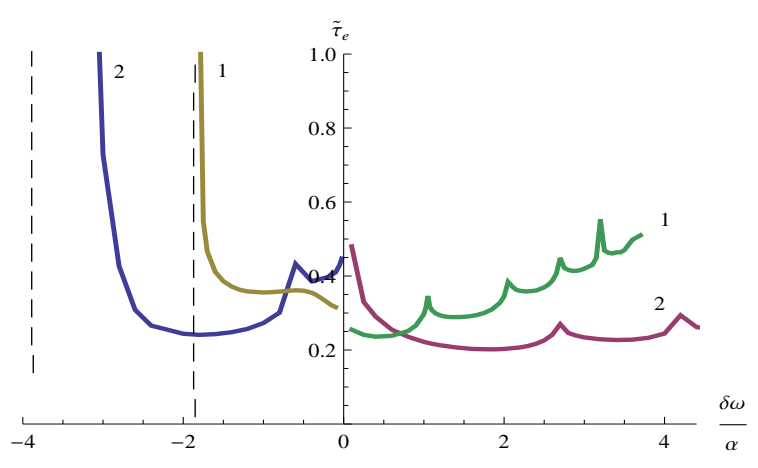

FIG. 3: Dependence of $\tilde{\tau}_{e}$ vs. $\delta \omega / \alpha$. Solution of eqs. (26) for the principal unstable triad $U_{2,-1}(\alpha=20.5)$, in two different sets of the initial data, 1 and 2: $1 .-\tilde{\tau}=0: a_{0}=0.05, d a_{0} / d \tilde{\tau}=$ $0.5 \alpha, \rho=0.4, \phi=\pi / 4 ; 2 .-\tilde{\tau}=0: a_{0}=0.05, d a_{0} / d \tilde{\tau}=0$, $\rho=0.4, \phi=\pi / 4$. The vertical dashed straightlines depict the mismatch thresholds for initial data 1 . and $2 .: \delta \omega_{t h} / \alpha \approx$ -1.85 and $\delta \omega_{t h} / \alpha \approx-3.9$, respectively.

\section{B. Stable triads $\left(\sigma_{1}=+1\right)$}

The amplitudes of the stable triad $S_{1,1}$ remain bounded at all times and exhibit irregular periodic oscillations. This may be seen in Fig. 4 where a saturation of the MRI is demonstrated for numerical solutions of eqs. (22)-(23).

\section{DISCUSSION AND SUMMARY}

Is is shown that at resonance conditions the weaklynonlinear evolution of unstable triads formed by the background MRI mode (first unstable slow AC mode) together with the associated two linearly stable MS and another slow AC modes can give rise to EMRI in the 


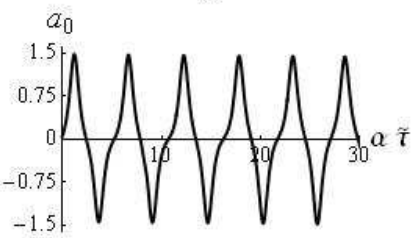

(b)

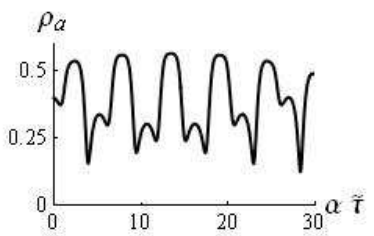

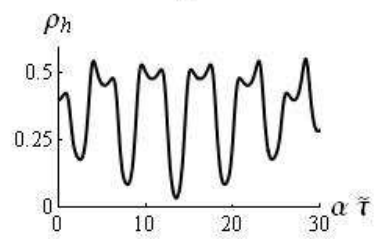

FIG. 4: Solution of eqs. (22)-(23) in the partial initial data $\left(\tilde{\tau}=0: a_{0}=0.05, d a_{0} / d \tilde{\tau}=0.5 \alpha, a_{1}=h_{1}=0.4\right)$ for the stable triad $S_{1,1}(\alpha=0.42, \delta \omega / \alpha=1)$.

vicinity of its linear stability threshold.

The triad amplitude relations for thin Keplerian disks are similar to those for other dispersive plasma media [17] 20], but exhibit some specific features. As was first noted in [18] for beam plasmas (see also detailed studies in [19] and [20]) zero-energy mode can excite EI in the vicinity of the linear stability threshold. Indeed, energy of the linear MRI is zero as was shown in [23]. As indicated in [18] -20], this results in the appearance of the second time derivative in the amplitude equation for zero-energy mode (see also [6]). Along with this there are some important distinctions between the two systems, among which the non-zero frequency mismatch is obligatory for EI in thin-disk plasmas, but it is not necessary for beam plasmas. In addition, the self-similarity laws for amplitudes of the triad in time in the blow-up point for beam plasmas [19] differ from those for thin-disk plasmas. In-spite of those differences the qualitative agreement between both systems is obvious. Also EMRI in thin disks either becomes less degenerous due to growth of the explosive time with increasing $|\Delta \omega|$, or, as in [17], saturated by turning into bounded oscillations for negative $\Delta \omega$ less than a threshold value (Fig. 3). Besides, as opposite to beam-plasma systems in [17] - 20] (for which $\Delta \omega$ assumed to be much smaller than the non-zero eigenfrequencies of all three interacting modes), for thin-disk plasmas the MRI unstable eigenmode has the zero-real frequency near the linear stability threshold, while $\Delta \omega$ should be of the order of small growth rate $\gamma$ of the MRI and much smaller than real eigenfrequencies of the rest two waves. Finally note that EMRI in thin disks is driven by the positive feedback between the MRI mode and the rest two waves. Under resonance conditions the positive feedback is provided by the force term in the Duffing equation for the MRI amplitude, where the force term grows and the MRI amplitude with it when amplitudes of the associated waves rise. In turn the amplitudes of the

associated waves rise with amplitude of the MRI mode. However for strict resonance conditions the positive feedback is degenerated. Indeed, amplitudes of the associated waves grow exponentially, but the feedback leaves the amplitude of the axisymmetric MRI mode bounded because of the force term constancy in time [8]. This degeneration of the positive feedback at $\delta \omega=0$ means that the presence of a small non-zero mismatch does not merely lead to small deviations from the strict-resonance case, but may change the behavior of the system in a fundamental way.

As physically evident EMRI should be saturated with time. Nonlinear terms with higher order harmonics in time neglected in the current approximation have been taken into account in a number of earlier studies in order to saturate EI (12 and references therein). However, this has been found ineffective by Mahoney 24] which shows that all higher order harmonics are of the same order and should be involved simultaneously in the blowup vicinity that rather requires solution of the compete nonlinear problem. It is suggested that the analytical results obtained in the present study will be useful for elucidating physical irregularities of MRI. It is conjectured that the EMRI describes an intermediate stage of the MRI nonlinear evolution during which a significant energy transfer takes place. That stage of the nonlinear interaction between the three resonant modes occurs before the blow-up and leads to the break-down of the present model. Direct numerical simulations can resolve the evolution beyond blow-up.

\section{Acknowledgments}

This work was supported by grant number 366/15 of the Israel Science Foundation.

\section{References}

[1] Velikhov E.P. Soviet Physics, JETP, 36:995 (1959).

[2] Chandrasekhar S. Proc. Natl. Acad. Sci., 46:353 (1960).

[3] Balbus S.A. and Hawley J.F. Astrophys. J., 376:214 (1991).

[4] Knobloch E. and Julien K. Phys. Fluids, 17:094106 (2005).

[5] Umurhan O.M., Menou K. and Regev O. Phys. Rev. Lett., 98:034501 (2007).

[6] Liverts E., Shtemler Y., Mond M., Umurhan O.M., and Bisikalo D.V. Phys. Rev. Lett., 109:224501 (2012).

[7] Shtemler Y., Liverts E. and Mond M. Phys. Rev. Lett., 111:231102 (2013).

[8] Shtemler Y., Mond M. and Liverts E. MNRAS, 439:1140 (2014). 
[9] Shtemler Y., Mond M. and Liverts E. MNRAS, 413:2957 (2011).

[10] Galeev A.A. and Oraevskii V.N. Soviet Phys. Doklady, 7:988 (1961-1962).

[11] Sagdeev R.Z. Galeev A.A. Nonlinear Plasma Theory W.A. Benjamin, New York, Amsterdam, 1969.

[12] Weiland J. Wilhelmson H. Coherent Nonlinear Interactions of Waves in Plasma Oxfod: Pergamon, 1977.

[13] Craik A.D.D. Wave Interactions and Fluid Flows Cambridge University Press, 1985.

[14] Dum C.T. and Sudan R.N. Phys. Rev. Lett., 23:1149 (1969).

[15] Rosenbluth M.N., Coppi B. Sudan R.N. 3rd Conf. on Plasma Phys. and Contr. Nucl. Fusion Res., Novosibirsk, 1968, 1, Paper CN 24/E13.
[16] Oraevski V. N. , Pavlenko V. P., Wilhelmson H., and Kogan E. Ya. Phys. Rev. Lett., 30:40 (1973).

[17] Fukai J., Krishan S., and Harris E. G. Phys. Rev. Lett., 23:910 (1969).

[18] Dum C.T. and Ott E. Plasma Phys. 13:177 (1971).

[19] Davydova T. A., Pavlenko V. P., Taranov V. B. and Shamrai K. P. Plasma Phys. 20:373 (1978).

[20] Pavlenko V. P. Plasma Phys. 20:771 (1978).

[21] Regev O. A \& A, 126:146 (1983).

[22] Shtemler Y., Mond M. and Liverts E. Astrophys. J., 665:1371 (2007).

[23] Khalzov I. V., Smolyakov A. I. and Ilgisonis V. I. Phys. Plasmas, 15:054501 (2008).

[24] Mahoney D. J. Stud Appl Math, 95:381 (1995). 